\title{
Leishmaniosis en un zorro (Cerdocyon thous) del Zoológico de la Ciudad de Corrientes (Argentina)
}

\author{
Burna, A.N.'; Imhoff, O. ${ }^{2}$; Montenegro, M.A.'; Sánchez Negrette, M.'; Catuogno, M.S. ${ }^{1}$
}

${ }^{1}$ Cátedra de Patología General y Sistemática, Facultad de Ciencias Veterinarias, Universidad Nacional del Nordeste, Sargento Cabral 2139, Corrientes (Argentina). ${ }^{2}$ Veterinario del Zoológico de la Ciudad de Corrientes. E-mail: patgral@vet.unne.edu.ar

\begin{abstract}
Resumen
Burna, A.N.; Imhoff, O.; Montenegro, M.A.; Sánchez Negrette, M.; Catuogno, M.S.: Leishmaniosis en un zorro (Cerdocyon thous) del Zoológico de la Ciudad de Corrientes (Argentina). Rev. vet. 26: 1, 75-78, 2015. En el continente americano existe una gran variedad de animales silvestres y domésticos implicados como reservorios de Leishmania sp. El perro doméstico desempeña un papel importante en la leishmaniosis visceral al conservar el vector en el ciclo urbano de la enfermedad. El objetivo del presente trabajo fue diagnosticar mediante síntomas clínicos y estudios serológico, histopatológico e inmunohistoquímico, un caso de leishmaniosis visceral en un "zorro de monte" (Cerdocyon thous) alojado en un zoo del nordeste argentino. Los estudios realizados permitieron confirmar el diagnóstico al constatarse la presencia del parásito en varios órganos. Se resalta la importancia de realizar pruebas diagnósticas para leishmaniosis antes de trasladar animales de zoológicos desde zonas endémicas hacia regiones consideradas libres de la enfermedad.
\end{abstract}

Palabras clave: zorro (Cerdocyon thous), Leishmania sp., histopatología, inmunohistoquímica, Argentina.

\begin{abstract}
Burna, A.N.; Imhoff O.; Montenegro M.A.; Sanchez Negrette, M; Catuogno M.S.: Leishmaniosis in a fox (Cerdocyon thous) from the Zoo of Corrientes City (Argentina). Rev. vet. 26: 1, 75-78, 2015. There are a variety of wild and domestic animals that have been implicated as reservoirs of Leishmania sp. in America. The domestic dog plays an important role as a reservoir of visceral leishmaniosis, allowing the urban cycle of the disease when the vector is present. The aim of this paper is to present a case of visceral leishmaniosis in a "forest fox" (Cerdocyon thous) by clinical symptoms together with serologic, histopathologic and inmunohistochemistry studies. The finds allowed to confirm the presence of the parasite in several organs. It is important to perform tests to diagnose leishmaniosis in zoo animals before transportation from endemic areas to those free of the disease.
\end{abstract}

Key words: fox (Cerdocyon thous), Leishmania sp., histopathology, immunohistochemistry, Argentina.

\section{INTRODUCCIÓN}

Las leishmanias son los protozoarios responsables de la leishmaniosis, enfermedad parasitaria que afecta al ser humano y animales domésticos, principalmente cánidos y especies silvestres (zorros, zarigüeyas, coatíes y roedores). En ocasiones se ha informado la existencia de esta enfermedad en animales silvestres o en especies salvajes en cautiverio, como zorros y lobos y primates. Cada especie del género Leishmania tiene uno o más reservorios primarios y puede infectar a otras especies.

Recibido: 4 noviembre 2014 / Aceptado: 16 diciembre 2014
En el continente americano existe una gran variedad de animales domésticos y de vida libre que han sido implicados como reservorios de leishmanias. En cautiverio, algunas especies silvestres cursan la infección en forma asintomática. En Brasil se ha reportado que algunos marsupiales y roedores de los géneros Proechimys y Oryzomys operan como reservorios de Leishmania amazonensis. El "perezoso de dos dedos" (Choloepus didactylus), así como el "oso melero" o "tamandua" (Tamandua tetradáctila) y otros marsupiales y roedores, pueden hospedar Leishmania guyanensis. Perros, equinos, mulas y roedores domésticos han sido reconocidos como reservorios de Leishmania braziliensis ${ }^{5,6}$. En América del Sur los cánidos son los reservorios más importantes de leishmanias ${ }^{7}$. 
En presencia del vector, el perro doméstico (Canis familiaris) desempeña un papel importante en la transmisión de la leishmaniosis visceral (LV), posibilitando el ciclo urbano de la enfermedad ${ }^{3}$. Muchas especies pueden actuar como reservorios del parásito, pero por lo general no son responsables de la transmisión a los seres humanos. Hay informes de infección en cánidos salvajes como el zorro de monte (Cerdocyon thous), el lobo de crin o "aguará guazú" (Chrysocyon brachyurus), el zorro canoso (Lycalopex vetulus) ${ }^{2,9}$ y el perro de monte (Spheotos venaticus) ${ }^{4}$.

Otros mamíferos silvestres como el agutí (Dasyprocta aguti), la comadreja overa (Didelphis albiventris), ratas silvestres (Cercomys cunicularius) y el ratón del arroz (Oryzomys eliurus), también pueden padecer LV tras la infección natural ${ }^{9}$. Sin embargo, en Brasil sólo $C$. thous es considerado como reservorio de esta enfermedad ${ }^{1}$, siendo discutible la importancia de otras especies silvestres ${ }^{3}$. Los cambios ambientales debido a la destrucción de los ecosistemas asociados a la migración de la población humana han favorecido la urbanización de la leishmaniosis ${ }^{3,8,10}$.

La presente comunicación tuvo como objetivo reportar para el nordeste argentino un caso de leishmaniosis visceral en un zorro de monte (Cerdocyon thous) que desarrolló síntomas característicos de la enfermedad, diagnóstico que fue confirmado mediante estudios serológico, histopatológico e inmunohistoquímico.

\section{MATERIAL Y MÉTODOS}

En marzo de 2013, un "zorro de monte", hembra, de 8 años de edad, alojado en el Zoológico de la Ciudad de Corrientes, Argentina, reveló zonas alopécicas en piel, así como pelo hirsuto a nivel de las caderas y en dorsal del carpo. Las lesiones fueron aumentando de tamaño y aparecieron úlceras en las zonas de apoyo, así como también onicogrifosis. El animal reveló un mal estado de nutrición, caquexia, atrofia muscular, conjuntivitis, crecimiento excesivo de uñas, dermatitis exfoliativa con descamación furfurácea de color plata, pelos hirsutos y ulceras cutáneas (Figura 1).

El paciente fue tratado con antibióticos de amplio espectro, desinfectantes y cicatrizantes, los cuales pro-

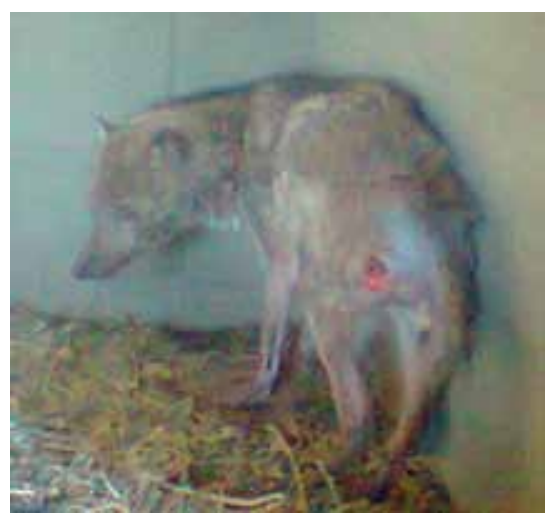

Figura 1. Se observa una lesión ulcerada en la región posterior de la cadera del zorro. dujeron una disminución en el tamaño de las lesiones, sin llegar a curarlas. Posteriormente fue trasladado a una reserva ecológica ubicada fuera de la ciudad y alojado en un recinto junto a otro zorro de monte clínicamente sano.

Con el fin de confirmar o descartar la presencia de Leishmania sp. se le extrajo sangre venosa a partir de cortes de uñas y se realizó punción con aguja fina e impronta de los bordes de las lesiones ulceradas, materiales que fueron enviados al laboratorio para realizar el diagnóstico parasitológico directo y serológico. Para este último se utilizó el test rápido para la detección de anticuerpos anti Leishmania sp. (Kalazar Detect Tm, Lab.InBios) específico para el complejo Leishmania donovani.

Tiempo después se produjo la muerte del animal, tras la cual se efectuó necropsia y extracción de muestras de órganos que fueron fijadas en formol bufferado al $10 \%$ para su estudio histopatológico e inmunohistoquímico. Las muestras fueron procesadas mediante la técnica histológica clásica para bloques parafinados, cortadas a $5 \mu \mathrm{m}$ y coloreados con hematoxilina y eosina. Para la técnica inmunohistoquímica, la inhibición de la peroxidasa endógena se llevó a cabo con peróxido de hidrógeno y metanol. La recuperación antigénica se realizó en horno microondas con las muestras sumergidas en buffer citrato $\mathrm{pH}$ 6. Para evitar coloraciones inespecíficas se utilizó suero normal equino. Posteriormente las muestras fueron incubadas con anticuerpo primario policlonal anti Leishmania sp. en cámara húmeda durante $12 \mathrm{~h} \mathrm{a} 4^{\circ} \mathrm{C}$. Luego fueron incubadas durante $30 \mathrm{~min}$ con anticuerpo secundario biotinilado, y más tarde incubadas otros 30 min con solución de extravidina, para finalizar con la aplicación de diaminobencidina, contracoloración con hematoxilina, deshidratación de las muestras y montaje con bálsamo de Canadá natural.

\section{RESULTADOS}

La serología para la detección de anticuerpos específicos para el complejo L. donovani fue reactiva. El examen parasitológico directo realizado en muestras obtenidas de lesiones cutáneas ulceradas reveló múltiples estructuras compatibles con amastigotes de Leishmania sp. dentro de macrófagos. La necropsia no reveló manifestaciones macroscópicas dignas de consideración.

El examen histopatológico reveló la presencia de macrófagos y múltiples focos de infiltrado linfoplasmocitario característicos de esta enfermedad en hígado, bazo, riñones, corazón, intestino, vejiga y piel. El hígado reveló una marcada colestasis, con necrosis centrolobulillar y degeneración hidrópica y grasa y (Figura 2). En riñones se observó congestión y cilindros hialinos. En piel de zona de la cadera y en dorsal del carpo se observaron lesiones ulceradas con abundante infiltrado inflamatorio, que comprometieron tanto a la dermis profunda como superficial, así como presencia 


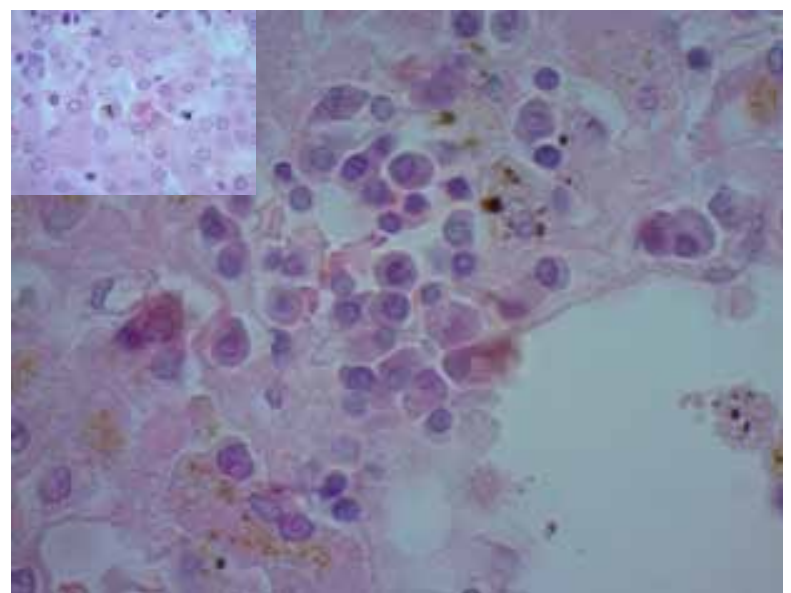

Figura 2. Hígado con infiltrado linfoplasmocitario y colestasis. En recuadro superior: macrófago conteniendo amastigotes de Leishmania sp. HE, 100x.

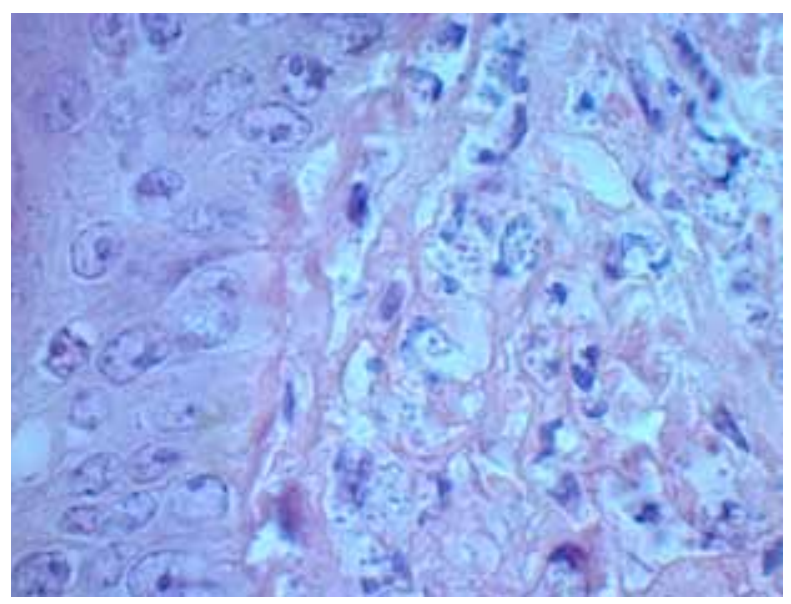

Figura 3. Piel. Dermis superficial con infiltrado inflamatorio y abundantes macrófagos conteniendo estructuras compatibles con amastigotes de Leishmania sp. HE, 100x.

de abundantes macrófagos con estructuras compatibles con amastigotes de Leishmania sp. en su interior (Figura 3). El músculo cardiaco presentó un leve infiltrado inflamatorio linfoplasmocitario.

La técnica inmunohistoquímica permitió demostrar la presencia de amastigotes dentro y fuera de los macrófagos. En el hígado se observaron amastigotes de Leishmania sp. en el epitelio de los conductos biliares y en macrófagos distribuidos entre el parénquima hepático. En intestino se observaron amastigotes dentro de macrófagos. En bazo se localizaron dentro y fuera de macrófagos. En piel se observaron dentro de macrófagos en la dermis y libres dentro de vasos linfáticos (Figura 4).

Para confirmar el diagnóstico clínico de leishmaniosis se considera importante realizar al menos dos pruebas de laboratorio: serología y examen parasitológico directo. Si bien el diagnóstico definitivo dependerá del reconocimiento de los amastigotes intracelulares, las técnicas inmunohistoquímicas permiten corroborar el diagnóstico parasitológico, revelando mayor sensibilidad y especificidad para la detección del parásito.

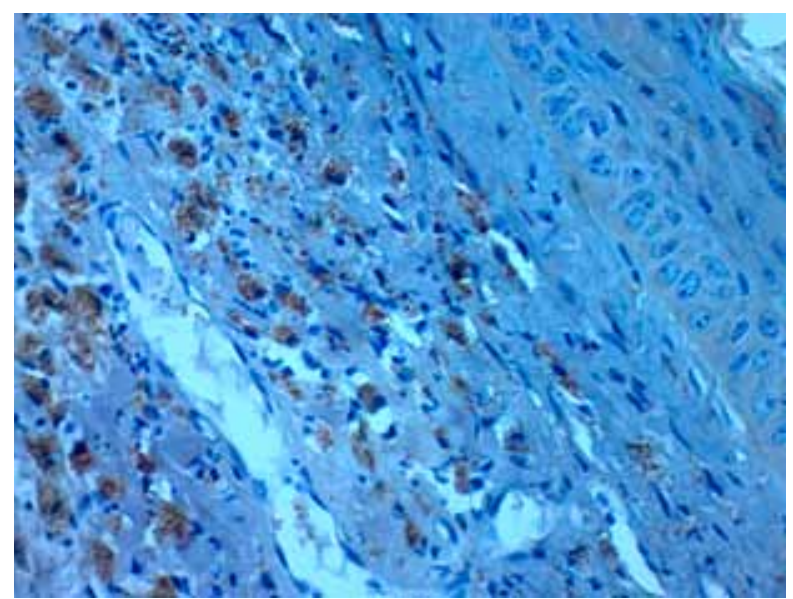

Figura 4. Piel. Dermis superficial con abundantes macrófagos con amastigotes de Leishmania sp. coloreados de color marrón, así como también amastigotes libres en vasos linfáticos dilatados. IHQ, 40x.

En conclusión, se confirma caso de leishmaniosis visceral en un zorro de monte alojado en un zoológico del nordeste argentino, siendo éste el primer caso diagnosticado por serología, histopatología e inmunohistoquímica en un animal silvestre en cautiverio con signos clínicos de leishmaniosis. Se resalta la importancia de realizar pruebas diagnósticas para leishmaniosis antes de trasladar animales de zoológicos desde regiones endémicas hacia zonas consideradas libres de la enfermedad.

\section{REFERENCIAS}

1. Courtenay O, Santana EW, Johnson PJ, Vasconcelos LA, Vasconcelos AW. 1996. Visceral leishmaniasis in the hoary zorro Dusicyon vetulus: a case of mistaken identity. Trans R Soc Trop Med Hyg 90: 498-502.

2. Curi NH, Miranda I, Talamoni SA. 2006. Serologic evidence of Leishmania infection in free-ranging wild and domestic canids around a Brazilian National Park. Mem Inst Oswaldo Cruz 101: 99-101.

3. Diniz SA, Silva FL, Carvalho AV, Bueno R, Guerra RM, Abreu AL, Santos RL. 2008. Animal reservoirs for visceral leishmaniasis in densely populated urban areas. $J$ Infect Dev Countries 2: 24-33.

4. Figueiredo FB, Gremião ID, Pereira SA, Fedulo LP, Menezes RC, Balthazar DA, Schubach TM, Madeira MF. 2008. First report of natural infection of a bush dog (Speothos venaticus) with Leishmania chagasi in Brazil. Trans R Soc Trop Med Hyg 102: 200-201.

5. Ministerio de Salud de Brasil. 1991. Guía de control de la leishmaniasis tegumentaria americana. Fundación Nacional de Salud. On line: www.monografias.com/tra- bajos89/prevalencia-factores-riesgo-leishmaniosis/prevalencia-factores-riesgo-leish- maniosis3.shtml

6. Ministerio de Salud de Brasil. 1996. Leishmaniasis tegumentaria americana en el Brasil. Cuaderno Informativo destinado a los trabajadores de salud. Fundación Nacional de Salud. On line: sisbib.unmsm.edu.pe/bvrevistas/dermatologia/v14_n2/pdf/a02.pdf 
7. Palatnik CB, Santos WR, França JC, Costa RT, Reis AB, Palatnik M, Mayrink W, Genaro O. 2001. Impact of canine control on the epidemiology of canine and human visceral leishmaniasis in Brazil. Am J Trop Med Hyg 65: 510-517.

8. Patz JA, Graczyk TK, Geller N, Vittor AY. 2000. Effects of environmental change on emerging parasitic diseases. Int J Parasitol 30: 1395-1405.
9. Sherlock IA. 1996. Ecological interactions of visceral leishmaniasis in the state of Bahia, Brazil. Mem Inst Oswaldo Cruz 91: 671-683.

10. Silva ES, Gontijo CM, Pacheco RS, Fiuza VO, Brazil RP. 2001. Visceral leishmaniasis in metropolitan region of Belo Horizonte state of Minas Gerais, Brazil. Mem Inst Oswaldo Cruz 96: 285-291. 